\title{
Wireless Charging Device for Artificial Cardiac Pacemaker
}

\author{
Quan Xiong ${ }^{1, a^{*}}$ \\ ${ }^{1}$ School of Automation Science and Electrical Engineering, Beihang University, Beijing 100191 \\ axiong_quan1991@yeah.net
}

Keywords: wireless power transfer; magnetic-coupled; cardiac pacemaker; battery charging

Abstract. To overcome the limitation of energy supply for artificial cardiac pacemaker, this paper investigated a wireless charging device based on the magnetic coupling resonant wireless transfer (MCR-WPT) technique. We designed the bilayer coupled coils for energy transmission, which had twice power factor than monolayer coils. The frequency is $200 \mathrm{kHz}$ when the MCR-WPT system was in resonance. Furthermore, we put the titanium alloy case behind the receiver coil to simulate the environment of artificial cardiac pacemaker. At last, we have done the charging experiment.

\section{Introduction}

The cardiac pacemaker has benefit millions of patients until now, however, energy supply has always been the main limitation to its application. The magnetic coupling resonant wireless power transfer (MCR-WPT) technique is based on an induced magnetic field, operating under the condition of strongly coupled magnetic resonance between two coils in midrange transmission [1]. The MCR-WPT technique with chargeable lithium-ion battery could well solve the problem of energy supply for cardiac pacemaker and avoid the postoperative infection. Currently, the study of wireless power transfer in implantable device concentrates on coil optimization design [2-4], frequency optimization selection [5-7], and circuit design [8-10] and so on. However, the study in energy supply for artificial cardiac pacemaker has been rarely investigated and most of people utilize receiver coil to replace battery directly powering the pacemaker [11], while the experimental subjects are often mice and it could not apply in the humanity [12].

In this paper, we investigated a wireless charging device for artificial cardiac pacemaker based on the MCR-WPT technique, applying the bilayer coil design to make two coils coupling stronger, and the charging experiment has been done to test the device.

\section{Coil design}

The transmitter and receiver coils are the key part of the whole device, which decide whether the system operates well. To make the two coils coupled stronger, we designed the bilayer coil. Compared to the monolayer coil, the bilayer coil has fourfold inductance and only twice resistance than the monolayer coil. Then the bilayer coil has twice power factor than the monolayer coil, thus the coupling of the two coils elevated. The two coils are made by printed circuit board (PCB), so their inductances are stable and easier to compensate. The transmitter coil is the regular plane circle design, as shown in Fig. 1(a), while the receiver coil needs to fit the surface of the pacemaker, so its shape is irregular, as shown in Fig. 1(b). Furthermore, the receiver coil applies the special flexible print circuit, which can bend in $360^{\circ}$, so it can better fit the surface of the pacemaker. 


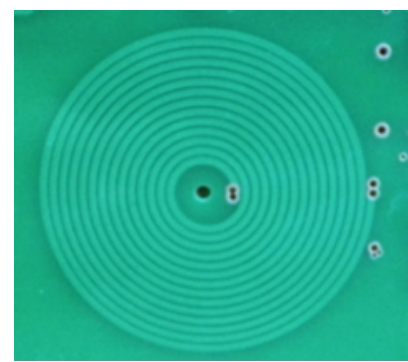

(a) Transmitter coil

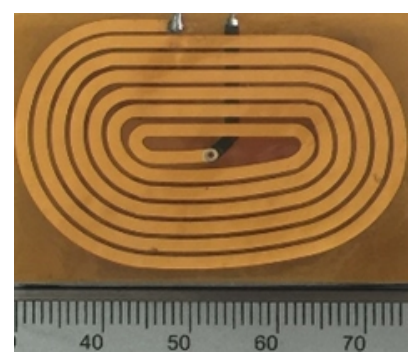

(b) Receiver coil

Fig. 1 The coupled two coils

\section{Circuit design}

The circuit of the wireless charging device are shown in Fig. 2. The primary and secondary coils are separated by an $8 \mathrm{~mm}$ air gap. The whole device consists of four parts. The first part is energy source, containing a signal generator and a signal amplifier, which provides a $200 \mathrm{kHz}$ sinusoidal voltage to power the system. The second part are the coupled coils and their compensating circuit, the system was in series-parallel compensating model, this model is easy to tune and the secondary part can get higher voltage. The third part consists of a schottky diode and a zener diode for rectifying and being a 5V DC power source for the last part. The last part is the charging circuit achieved by the IC SLM4900 and its application circuit, which can charge a single 4.2V lithium-ion battery. The resistance $R_{\text {set }}$ can adjust the charging current, and maximum is $800 \mathrm{~mA}$.

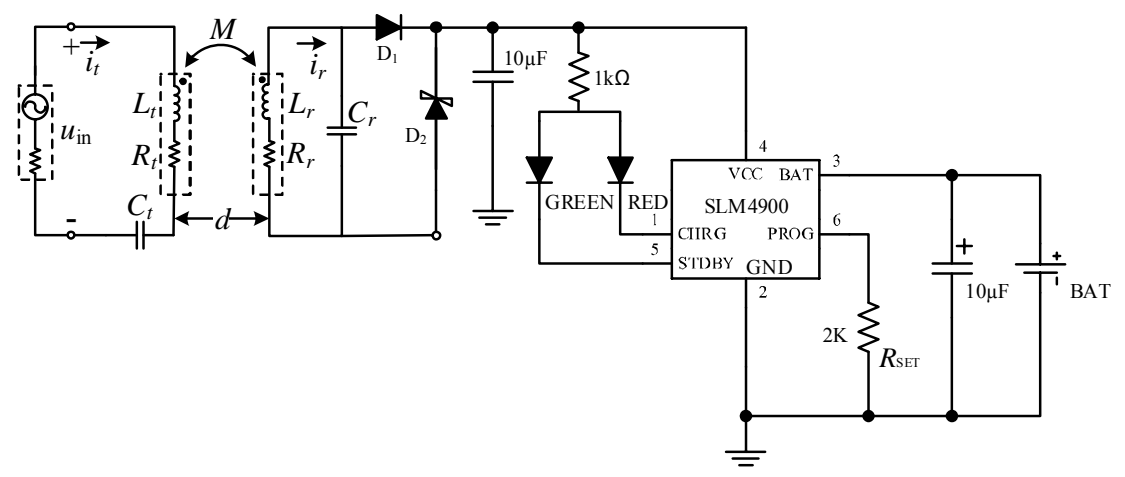

Fig. 2 circuit of the wireless charging device

\section{Battery selection}

Considering the heat of the coils and the psychological states of patients, the charging process should not last too long. Actually, 30 minutes could be suitable, so the capacity of the lithium-ion battery should be chosen considerately. From the data [13], the 0.92Ah lithium battery for single-chamber pacemaker could last 10.4 years and the 1.3Ah lithium battery for dual-chamber pacemaker could last 11.3 years. So we chose a $1050 \mathrm{mAh}$ lithium-ion battery at last, and we plan to charge the battery when its remaining power decreases to $80 \%$. The battery longevity is shown in the equation (1),

$$
L=Q_{d e l} / 8766 I
$$

$I$ is the current output of the pacemaker, usually $10 \mu \mathrm{A}[14], L$ is the battery longevity. From the equation (1), $20 \%$ power of our battery can last 2.39 years. 
In the wireless charging device, we set the charging current $450 \mathrm{~mA}$. To charge the battery from $80 \%$ to $100 \%$, it needs about 28 minutes, meeting our requirements.

\section{Charging experiment}

In the charging experiment, we put a $31.4 \mathrm{~mm} \times 47.4 \mathrm{~mm} \times 8.1 \mathrm{~mm}$ titanium alloy case behind the receiver coil to simulate the environment of pacemaker, as shown in Fig. 3(a), and the distance between the receive coil and titanium alloy case was $4 \mathrm{~mm}$, the distance between the transmission coil and titanium case was $12 \mathrm{~mm}$. The whole device is shown in Fig. 3(b).

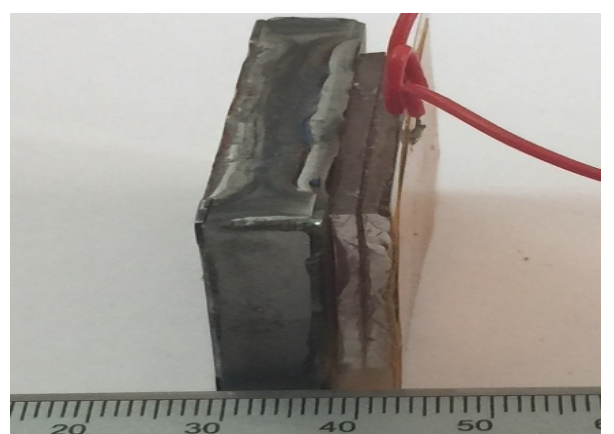

(a)

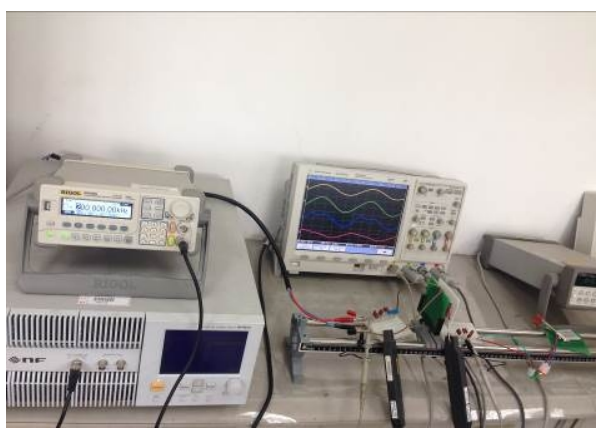

(b)

Fig. 3 the wireless charging device

The frequency of power source was $200 \mathrm{kHz}$, and the original voltage of lithium-ion battery was $3.9912 \mathrm{~V}$. Measured in the experiment, the input voltage $u_{\text {in }}$ was $26.3 \mathrm{~V}$ (peak-peak), the input current $i_{\text {in }}$ was $4.7 \mathrm{~A}$ (peak-peak). The charging curve is shown in Fig. 4, the whole process lasted 32 minutes and the voltage of battery increased from $3.9912 \mathrm{~V}$ to $4.2060 \mathrm{~V}$, reaching the expectation.

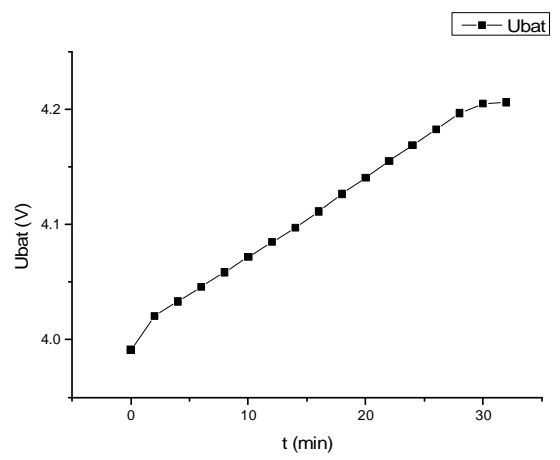

Fig. 4 the charging curve

\section{Conclusion}

This paper investigated the wireless charging device for artificial cardiac pacemaker based on the magnetic coupling resonant wireless transfer technique. The bilayer coil design achieved stronger coupling and stable energy transmission. The titanium alloy case simulated the actual environment that made the statistics more reliable. Furthermore, the experiment achieved charging the battery from $3.9912 \mathrm{~V}$ to $4.2060 \mathrm{~V}$ in 32 minutes, reaching our expectation and fulfilling the demand of power supply for artificial cardiac pacemaker.

\section{Reference}

[1] A. Kurs, A. Karalis, R. Moffatt, J. D. Joannopoulos, P. Fisher, and M. Soljacic, "Wireless 
Power Transfer via Strongly Coupled Magnetic Resonances," Science, vol. 317, no. 5834, pp. 83-86, Jul. 2007.

[2] A. K. RamRakhyani and G. Lazzi, "On the Design of Efficient Multi-Coil Telemetry System for Biomedical Implants," IEEE Transactions on Biomedical Circuits and Systems, vol. 7, no. 1, pp. 11-23, 2013.

[3] R. Wu, S. Raju, M. Chan, J. K. O. Sin, and C. P. Yue, "Silicon-Embedded Receiving Coil for High-Efficiency Wireless Power Transfer to Implantable Biomedical ICs," IEEE Electron Device Letters, vol. 34, no. 1, pp. 9-11, 2013.

[4] S. Kim, J. S. Ho, and A. S. Y. Poon, "Wireless Power Transfer to Miniature Implants: Transmitter Optimization," IEEE Transactions on Antennas and Propagation, vol. 60, no. 10, pp. 4838-4845, 2012.

[5] H. Jiang, J. Zhang, D. Lan, K. K. Chao, S. Liou, H. Shahnasser, R. Fechter, S. Hirose, M. Harrison, and S. Roy, "A Low-Frequency Versatile Wireless Power Transfer Technology for Biomedical Implants," IEEE Transactions on Biomedical Circuits and Systems, vol. 7, no. 4, pp. 526-535, 2013.

[6] A. S. Y. Poon, S. O’Driscoll, and T. H. Meng, “Optimal Frequency for Wireless Power Transmission Into Dispersive Tissue," IEEE Transactions on Antennas and Propagation, vol. 58, no. 5, pp. 1739-1750, 2010.

[7] R.-F. Xue, K.-W. Cheng, and M. Je, "High-Efficiency Wireless Power Transfer for Biomedical Implants by Optimal Resonant Load Transformation," IEEE Transactions on Circuits and Systems I: Regular Papers, vol. 60, no. 4, pp. 867-874, Apr. 2013.

[8] X. Li, C.-Y. Tsui, and W.-H. Ki, "A $13.56 \mathrm{MHz}$ Wireless Power Transfer System With Reconfigurable Resonant Regulating Rectifier and Wireless Power Control for Implantable Medical Devices," IEEE Journal of Solid-State Circuits, vol. 50, no. 4, pp. 978-989, Apr. 2015.

[9] H.-K. Cha, W.-T. Park, and M. Je, "A CMOS Rectifier With a Cross-Coupled Latched Comparator for Wireless Power Transfer in Biomedical Applications," IEEE Transactions on Circuits and Systems II: Express Briefs, vol. 59, no. 7, pp. 409-413, Jul. 2012.

[10]D. Ahn and S. Hong, "Wireless Power Transmission With Self-Regulated Output Voltage for Biomedical Implant," IEEE Transactions on Industrial Electronics, vol. 61, no. 5, pp. 2225-2235, 2014.

[11] Shuenn-Yuh Lee, Cheng-Han Hsieh, and Chung-Min Yang, "Wireless Front-End with Power Management for an Implantable Cardiac Microstimulator," IEEE Transactions on Biomedical Circuits and Systems, vol. 6, no. 1, pp. 28-38, Feb. 2012.

[12] J. I. Laughner, S. B. Marrus, E. R. Zellmer, C. J. Weinheimer, M. R. MacEwan, S. X. Cui, J. M. Nerbonne, and I. R. Efimov, "A Fully Implantable Pacemaker for the Mouse: from Battery to Wireless Power," PLoS ONE, vol. 8, no. 10, p. e76291, 2013.

[13] Information on http://www.vitatron.com/ous/physician.html.

[14]J. G. Webster, Medical Instrumentation Application and Design, 4th.ed. New York: Wiley, 2010. 